\title{
La cathédrale Saint-Étienne d'Auxerre : résultats récents des recherches pluridisciplinaires et internationales
}

\section{Christian Sapin}

\section{(2) OpenEdition \\ 1 Journals}

\section{Édition électronique}

URL : https://journals.openedition.org/cem/6902

DOI : $10.4000 /$ cem.6902

ISSN : 1954-3093

Éditeur

Centre d'études médiévales Saint-Germain d'Auxerre

\section{Édition imprimée}

Date de publication : 15 août 2008

ISSN : 1623-5770

Référence électronique

Christian Sapin, « La cathédrale Saint-Étienne d'Auxerre : résultats récents des recherches

pluridisciplinaires et internationales », Bulletin du centre d'études médiévales d'Auxerre / BUCEMA [En

ligne], 12 | 2008, mis en ligne le 29 juillet 2008, consulté le 22 septembre 2022. URL : http:// journals.openedition.org/cem/6902; DOI : https://doi.org/10.4000/cem.6902

Ce document a été généré automatiquement le 22 septembre 2022.

\section{(c) (i) (2) (2)}

Creative Commons - Attribution - Pas d'Utilisation Commerciale - Partage dans les Mêmes Conditions 4.0 International - CC BY-NC-SA 4.0

https://creativecommons.org/licenses/by-nc-sa/4.0/ 


\title{
La cathédrale Saint-Étienne d'Auxerre : résultats récents des recherches pluridisciplinaires et internationales
}

\author{
Christian Sapin
}

1 La tenue à Auxerre du colloque international autour des travaux sur la cathédrale a été un moment important de nos rencontres scientifiques de 2007. Ce fut un succès par le nombre de présents - plus d'une centaine durant trois journées - et par les échanges fructueux qui ont eu lieu entre les participants. La prochaine publication des actes est toujours envisagée avec le CTHS. Elle devrait permettre de garder un témoignage des travaux présentés, qui, rappelons-le, devaient synthétiser et mettre en perspective les nombreuses données nouvelles accumulées au cours des sept années d'observations pendant le chantier de restauration. Cette longue entreprise constitue sans doute un des rares exemples en France de ce qu'il est possible de faire et de dire encore à partir d'un ensemble cathédral.

2 Les propos historiques ou généraux tenus en premier lieu par Vincent Tabbagh puis dans la table ronde par Alain Rauwel, Guy Lobrichon, Nicolas Reveyron ou Dominique Iogna-Prat ont placé d'emblée la cathédrale comme un espace à part qui ne répondait pas à un usage purement fonctionnaliste et encore moins à une construction de tout un peuple comme l'historiographie depuis le XIX siècle avait tendance à nous le laisser croire. Il nous faut changer notre image de la cathédrale et l'iconographie présentée par Denis Cailleaux a bien montré comment la perception du lieu avait changé dans sa simple représentation.

3 Un des enjeux de cette rencontre était la nouvelle chronologie proposée par plusieurs acteurs de terrains, qui, à la suite de l'étude des toitures (Sylvain Aumard) et de la charpente (Catherine Lavier, Christine Locatelli et Didier Pousset), avaient, à plusieurs reprises au cours de ces dernières années, réuni les uns et les autres autour d'Harry Titus, Dieter Kimpel et Heike Hansen. Pour une fois, la connaissance des matériaux 
n'était pas séparée de l'histoire. Les arguments historiques ont trouvé des échos dans les techniques et inversement. La présence importante du métal (Sylvain Aumard, Philippe Dillmann et Maxime L'Héritier) en différents points de la cathédrale, dans des phases désormais bien datées du XIV ${ }^{\mathrm{e}}$ siècle, fournit ainsi, par exemple, des avancées substantielles sur le progrès des procédés de fabrication. De même, les relevés précis de l'équipe de Stuttgart (université et crédits DFG) pour les façades, alliées à la connaissance de la pierre utilisée (Stéphane Büttner et Lise Leroux) ont conduit les historiens de l'art à réfléchir différemment sur la mise en place et donc à la chronologie de la sculpture des portails avec des ateliers importants, notamment au XIV ${ }^{\mathrm{e}}$ siècle. La place des portails ouvre en même temps sur les choix iconographiques pratiqués pour le portail central ou les portails latéraux. De même que les verrières étudiées par Françoise Perrot, Sylvie Balcon et Isabelle Baudoin ou les peintures murales (MarieGabrielle Caffin) révélaient d'autres affinités. Ces mises en perspectives des matériaux comme du décor trouveront d'amples développements dans la publication avec Peter Kurmann, Fabienne Joubert, Annaïg Chatain et Dany Sandron. Les espaces propres à la cathédrale depuis la crypte romane conservée (Christian Sapin) jusqu'aux nouvelles chapelles enfin présentées (Kritina Krüger) ou la chapelle gothique Notre-Dame des Vertus, dont les traces ont été retrouvées lors de la restauration de la façade ouest, en passant par le chœur et la nef, ont gagné en compréhension, cohérence et chronologie. La cathédrale n'est pas réduite à quelques nouvelles formes inventives mais devient une entreprise infinie, où chaque élément renvoie, pour peu qu'on sache l'interroger, à un ensemble construit et à une histoire continue. La transmission de ce bien de l'Histoire, sous la responsabilité aujourd'hui de l'architecte en chef des Monuments historiques Bruno Decaris et de la Ville d'Auxerre, propriétaire, est devenu le souci de tous.

Rappel du programme

Bruno DECARIS, Le chantier moderne, à l'origine de la recherche actuelle

5 Vincent TABBAGH, La cathédrale, un bien commun

6 Denis CaIlleaux, Auxerre : les images de la cathédrale

7 Présentation collective, Une nouvelle chronologie établie à partir des observations et relevés faits pendant les travaux [avec commentaire de posters]

Le chantier

Christian SAPIN, À l'origine du chantier gothique : la crypte ( $\mathrm{V}^{\mathrm{e}}-\mathrm{XI}{ }^{\mathrm{e}}$ siècle)

Dieter KIMPEL, L'architecture gothique et les observations sur le chœur d'Auxerre

Harry Titus, La problématique de construction du chœur

Heike HANSEN, Observations sur la structure du transept

Kristina KRÜGER, Les chapelles de la cathédrale (chronologie, fondation et aménagement)

Dany SANDRON, Analyse de la structure de la façade occidentale

Les matériaux du bâti

Sylvain AUMARD, Nouvelles données sur le chantier des toitures

Catherine LAVIER, Didier Pousset et Christine LocATELI, Les charpentes de la cathédrale comme référentiel de dendrochronologie et perspectives sur l'approvisionnement en bois 
Stéphane BÜTTNER et Lise LEROUX, Les pierres de la cathédrale : l'approvisionnement du chantier

Sylvain AUMARD et Maxime L'HeRITIER, Le métal dans l'architecture de la cathédrale La sculpture

Heike HANSEN, Présentation générale de la sculpture

Harry Titus, Observations sur les têtes sculptées du déambulatoire du chœur

Peter KuRmann, Les portails de saint Étienne aux cathédrales de Paris, Meaux et Auxerre : analyse comparative

Marcello ANGHEBEN, L'iconographie du Jugement dernier

Fabienne JOUBERT, Le portail central : les voussures

Annaïg CHATAIN, Le portail du bras nord du transept

La peinture murale et le vitrail

Marie-Gabrielle CAfFIN, La place de la peinture murale dans le programme gothique de la cathédrale

Isabelle BAUDOIN, Observations et étude des vitraux de la nef

Françoise PERROT, Les verrières basses du chœur: observations et perspective de recherche

Sylvie BALCON, Les verrières hautes du chœur : étude et perspective de recherche

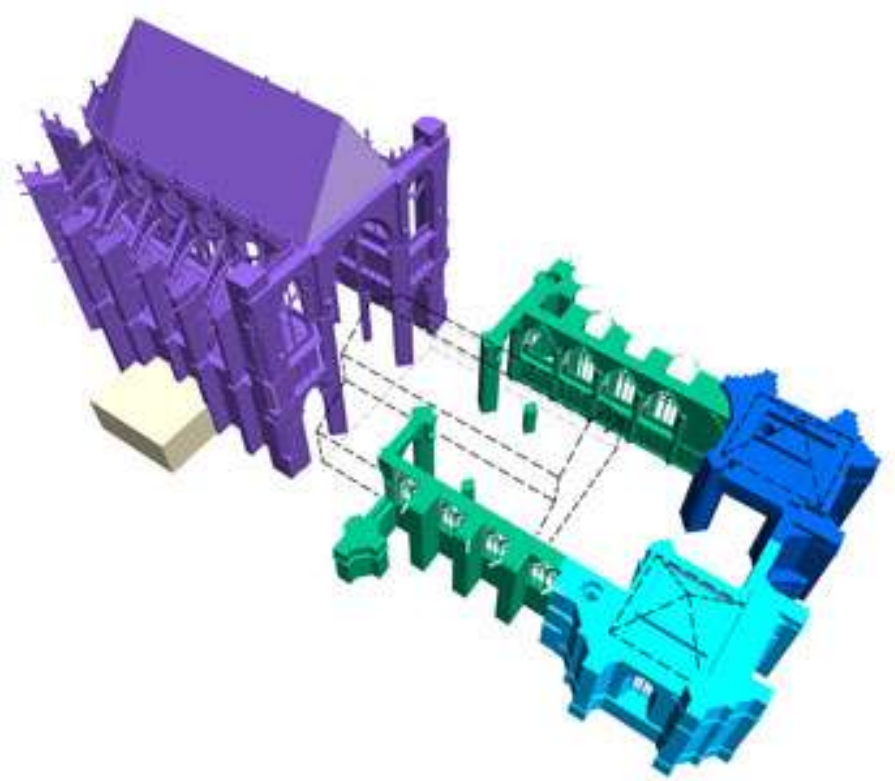

Fig. 1 - Cathédrale Saint-Étienne, chantier gothique vers 1320 (restitution, modélisation G. Etchenacher, université de Stuttgart). 
INDEX

Index géographique : France/Auxerre

Mots-clés : cathédrale, Saint-Etienne d'Auxerre 